\title{
Inducible Clindamycin Resistance in Methicillin-Resistant and-Susceptible Staphylococcus aureus Isolated From South East of Iran
}

\author{
Shahla Mansouri ${ }^{1} ;$ Javid Sadeghi ${ }^{1,2^{*}}$ \\ ${ }^{1}$ Department of Microbiology, Kerman University of Medical Sciences, Kerman, IR Iran \\ ${ }^{2}$ Department of Microbiology, Tabriz University of Medical Sciences, Tabriz, IR Iran \\ *Corresponding author: Javid Sadeghi, Department of Microbiology, Tabriz University of Medical Sciences, Tabriz, IR Iran. Tel/Fax: +98-4133364661, E-mail: javid4888@yahoo.com
}

Received: May 08, 2013; Revised: September 21, 2013; Accepted: November 30, 2013

\begin{abstract}
Background: Clindamycin is a frequently used antimicrobial therapeutic medicine used for the treatment of skin and soft tissue infections caused by Staphylococcus aureus strains. Resistance to this antibiotic is either constitutive or inducible. Constitutive resistance to clindamycin could be detected by standard susceptibility testing methods. Inducible clindamycin resistance could not be detected by in vitro routine tests. This type of resistance can be identified by D-test.

Objectives:The outbreak of inducible resistance to clindamycin in methicillin resistant and-susceptible S. aureus isolates were investigated in this study.

Materials and Methods: Totally 162 S. aureus isolates were evaluated for inducible clindamycin resistance by D-test in accordance with clinical and laboratory standards institute (CLSI) guidelines.

Results: Inducible clindamycin resistance was detected in $8.64 \%$ of $S$. aureus isolates. Inducible and constitutive resistance to clindamycin was found to be higher in methicillin resistant S. aureus ( $11.95 \%$ and $47.8 \%$ respectively) compared to methicillin susceptible S. aureus ( $4.28 \%$ and $2.85 \%$ respectively) isolates.

Conclusions: Our results showed that inducible resistance to clindamycin in S. aureus isolates is relatively higher in this region. Therefore, D-test should be performed to prevent treatment failures against infections caused by $S$. aureus, which are resistant to erythromycin and the sensitive ones against clindamycin.
\end{abstract}

Keywords:Clindamycin; Staphylococcus aureus; D-test, Inducible Resistance

\section{Background}

Macrolide, lincosamide and streptogramin B (MLSB) antibiotics are structurally different with a same mechanism of action. These drugs, especially clindamycin, are alternative drugs for some Staphylococcus aureus infections such as skin and soft tissue infections particularly in penicillin-allergic patients $(1,2)$. Clindamycin resistance in $S$. aureus strains may be either constitutive (cMLSB) or inducible (iMLSB). Inducible resistance to clindamycin was first recognized in the early 1960 s. Relapse of infection in a patient with S. aureus endocarditis was reported in 1976 (3). Detection of isolates with inducible clindamycin resistance is a problematic issue for the routine diagnosis, because they are erythromycin resistant and clindamycin susceptible in vitro and in the condition that they are not place adjacent to each other. In these cases, in vivo treatment with clindamycin may lead to clinical therapeutic failures $(4,5)$.

\section{Objectives}

The aim of this study was to determine the incidence of inducible clindamycin resistance in S. aureus isolates collected from clinical specimens in this geographic area.

\section{Materials and Methods}

Totally 162 S. aureus isolates were collected from clinical specimens of patients admitted to three university hospitals with 462, 420 and 300 bed capacities in Kerman, south-east of Iran, from March 2011 to February 2012. Confirmation of S. aureus isolates and detection of methicillin resistant S. aureus (MRSA) were performed by phenotypic and genotypic methods as previously described (6). Only one isolate per patient was included in the study. 'D-test' was used for detection of inducible resistance to clindamycin. This test was performed by placing an erythromycin $(15 \mu \mathrm{g})$ disk (Mast, Group Ltd., Merseyside, UK) at a distance of 15 to $20 \mathrm{~mm}$ from clindamycin $(2 \mu \mathrm{g})$ disk (Mast, Group Ltd., Merseyside, UK) on a Mueller-Hinton agar plate inoculated with 0.5 McFarland standard bacterial suspensions. Following overnight incubation at $37^{\circ} \mathrm{C}$, flattening of the clindamycin zone adjacent to the erythromycin disk (D shaped) indicated inducible clindamycin resistance (7). S. aureus ATCC 25923 was used as the control strain.

\section{Results}

From 162 S. aureus isolates tested for determination of

Copyright (C) 2014,Ahvaz Jundishapur University of Medical Sciences; Published by Kowsar. This is an open-access article distributed under the terms of the Creative Commons Attribution-NonCommercial 4.0 International License (http://creativecommons.org/licenses/by-nc/4.0/) which permits copy and redistribute the material just in noncommercial usages, provided the original work is properly cited. 
inducible clindamycin resistance, 70 (43.2\%) were MSSA and $92(56.8 \%)$ isolates were MRSA (Table 1$)$. Susceptibility to erythromycin and clindamycin of these isolates is presented in Table 1 . Sensitivity to both erythromycin and clindamycin was significantly higher in MRSA compared to MSSA isolates. Resistance to methicillin, erythromycin and clindamycin was observed in 92 (56.8\%), 75 (46.29\%) and $46(28.4 \%)$ of the isolates, respectively. Inducible resistance to clindamycin was determined in 14 (8.64\%) isolates (D-test positive, Figure 1). Table 2 shows the distribution of D-test positive $S$. aureus isolates in clinical specimens. Among those, 11 (78.57\%) were MRSA and 3 (22.43\%) were MSSA (Table 2).

\section{Discussion}

Clindamycin is an effective antibiotic agent used in the treatment of skin and soft-tissue infections caused by $S$. aureus isolates. This antibiotic has good tissue penetration, potential antitoxin effects, and also accumulates in abscesses (8). However, resistance to clindamycin may develop in $S$. aureus isolates with inducible phenotype and these isolates have a high rate of spontaneous mutation which would enable these isolates to develop constitutive resistance to clindamycin during therapeutic process (4).

A positive D-test indicates the existence of inducible resistance to clindamycin. In this study $8.64 \%$ of $162 \mathrm{~S}$. aureus isolates were D-test positive that is comparable with the findings of the studies by Rahbar and Hajia (9.7\%) (9) and Sedighi et al. (6.3\%) (10) From Iran and Azap et al. (5.7\%) from Turkey (11). The prevalence of inducible and constitutive clindamycin resistance differs in different geographic areas (12).

In our study high number (46.29\%) of S. aureus isolates were resistant to erythromycin. Among those, 14 (18.66\%) isolates had positive and 15 (20\%) had negative D-test results and 46 (61.33\%) were constitutive clindamycin resistance. In this study the prevalence of inducible and constitutive resistance to clindamycin in MRSA (11.95\% and

Table 1. Susceptibility Patterns of Isolated S. aureus Against Erythromycin and Clindamycin ${ }^{\mathrm{a}, \mathrm{b}}$

\begin{tabular}{|c|c|c|c|}
\hline $\begin{array}{l}\text { Susceptibility Pattern } \\
\text { (Phenotype) }\end{array}$ & MSSA & MRSA & Total \\
\hline $\operatorname{Ery}^{S} \mathrm{Cli}^{\mathrm{S}}$ & $57(81.43)$ & $26(28.26)$ & $83(51.23)$ \\
\hline $\operatorname{Ery}^{\mathbf{r}} \mathrm{Cli}^{\mathbf{r}}\left(\mathrm{cMLS}_{\mathbf{B}}\right)$ & $2(2.85)$ & $44(47.82)$ & $46(28.39)$ \\
\hline $\begin{array}{l}\operatorname{Ery}^{\mathbf{r}} \mathrm{Cli}^{\mathrm{S}} \text { (D test positive, } \\
\text { iMLS }_{\mathbf{B}} \text { ) }\end{array}$ & $3(4.28)$ & $11(11.95)$ & $14(8.64)$ \\
\hline $\operatorname{Ery}^{\mathbf{r}} \mathrm{Cli}^{\mathrm{S}}$ (D test negative) & $5(7.14)$ & $10(10.87)$ & $15(9.26)$ \\
\hline $\operatorname{Ery}^{S} \mathrm{Cli}^{\mathbf{r}}$ & $2(2.85)$ & $1(1.08)$ & $3(1.85)$ \\
\hline Total & $70(43.2)$ & $92(56.8)$ & $162(100)$ \\
\hline \multicolumn{4}{|c|}{$\begin{array}{l}\text { a Abbreviations: MSSA, Methicillin susceptible S. aureus; MRSA, } \\
\text { Methicillin resistant S. aureus; Ery, Erythromycin; Cli, Clindamycin; } \\
\text { s, Sensitive, r, Resistant; cMLSB, Constitutive macrolide lincosamide } \\
\text { and streptogramin B; iMLSB, Inducible macrolide lincosamide and } \\
\text { streptogramin B. } \\
\text { b Data are presented as No.(\%). }\end{array}$} \\
\hline
\end{tabular}

Figure 1. Inducible Clindamycin Resistance (D-test)

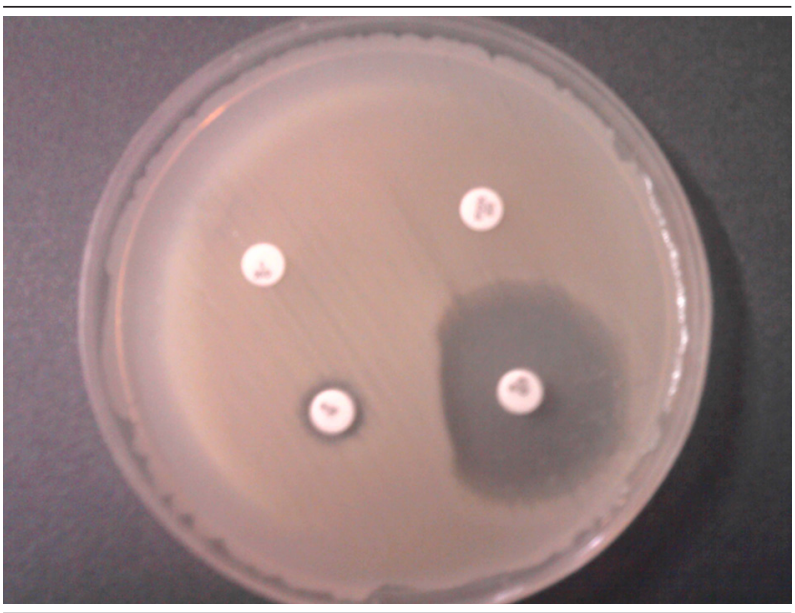

Flattening of the clindamycin zone adjacent to the erythromycin disk produces a D shape.

Table 2. Distribution of D-test Positive Isolates Collected From Clinical Specimens ${ }^{\mathrm{a}, \mathrm{b}}$

\begin{tabular}{|c|c|c|c|}
\hline \multirow[t]{2}{*}{ Isolated Area } & \multicolumn{3}{|c|}{ D-Test Positive } \\
\hline & MSSA & MRSA & Total \\
\hline Urine, $(n=45)$ & $1(2.22)$ & $6(13.33)$ & $7(15.55)$ \\
\hline Wound, $(\mathbf{n}=38)$ & $1(2.63)$ & $5(13.15)$ & $6(15.78)$ \\
\hline Bronco alveolar lavage, $(n=14)$ & $1(7.14)$ & 0 & $1(7.14)$ \\
\hline other samples $^{\mathrm{C}},(\mathrm{n}=65)$ & 0 & 0 & 0 \\
\hline Total, $(\mathrm{n}=162)$ & $3(1.85)$ & $11(6.8)$ & $14(8.64)$ \\
\hline
\end{tabular}

${ }^{\mathrm{a}}$ MSSA, Methicillin susceptible S. aureus; MRSA, Methicillin resistant $S$. aureus.

b Data are presented as No. (\%).

c Including: blood, skin and soft tissue, cerebrospinal fluid.

$47.8 \%$ respectively) is higher than those in MSSA ( $4.28 \%$ and $2.85 \%$, respectively). Our results were in accordance to the results of Seifi et al. (13) from Iran. The results of this study revealed that inducible resistance to clindamycin in S. aureus isolates is relatively high in this region. Since isolates with inducible resistance may mutate and change to constitutive resistance. Therefore, D-test should be performed to prevent treatment failures of $S$. aureus infections caused by the agents that are resistant to erythromycin and sensitive to clindamycin.

\section{Acknowledgements}

The authors wish to thank the authorities of Kerman University of Medical Sciences and in School of Medicine, for their support.

\section{Authors' Contributions}

Study concept and design: Mansouri, Sadeghi. Analysis and interpretation of data: Mansouri, Sadeghi. Drafting: Sadeghi. Critical revision of the manuscript for important intellectual content: Mansouri. 


\section{Funding/Support}

This study was supported by a grant (no 90/240) from Kerman University of Medical Sciences.

\section{References}

1. Drinkovic D, Fuller ER, Shore KP, Holland DJ, Ellis-Pegler R. Clindamycin treatment of Staphylococcus aureus expressing inducible clindamycin resistance. J Antimicrob Chemother. 2001;48(2):315-6.

2. Leclercq R, Courvalin P. Bacterial resistance to macrolide, lincosamide, and streptogramin antibiotics by target modification. Antimicrob Agents Chemother.1991;35(7):1267-72.

3. Woods CR. Macrolide-inducible resistance to clindamycin and the D-test. Pediatr Infect Dis J. 2009;28(12):1115-8.

4. Prabhu K, Rao S, Rao V. Inducible Clindamycin Resistance in Staphylococcus aureus Isolated from Clinical Samples.J Lab Physicians. 2011;3(1):25-7.

5. Rybak MJ, LaPlante KL. Community-associated methicillinresistant Staphylococcus aureus: a review. Pharmacotherapy. 2005;25(1):74-85.

6. Sadeghi J, Mansouri S. Molecular characterization and antibiotic resistance of clinical isolates of methicillin-resistant Staphylo- coccus aureus obtained from Southeast of Iran (Kerman). APMIS 2014;122(5):405-11.

7. Clinical and Laboratory Standards Institute. M02-A10 Performance standards for antimicrobial disk susceptibility tests; approved standard-tenth edition. 2009.

8. Patel M, Waites KB, Moser SA, Cloud GA, Hoesley CJ. Prevalence of inducible clindamycin resistance among community- and hospital-associated Staphylococcus aureus isolates. J Clin Microbiol. 2006;44(7):2481-4.

9. Rahbar M, Hajia M. Inducible clindamycin resistance in staphylococcous aureus. PakJ Biol Sci. 2007;10(1):189-92.

10. Sedighi I, Yousefi Mashouf R, Pak N, Seif Rabiee MA. D-Test Method for Detection of Inducible Clindamycin Resistance in Staphylococcus aureus. Iran J Pediatr. 2009;19(3):293-7.

11. Azap OK, Arslan H, Timurkaynak F, Yapar G, Oruc E, Gagir U. Incidence of inducible clindamycin resistance in staphylococci: first results from Turkey. Clin Microbiol Infect. 2005;11(7):582-4.

12. Fiebelkorn KR, Crawford SA, McElmeel ML, Jorgensen JH. Practical disk diffusion method for detection of inducible clindamycin resistance in Staphylococcus aureus and coagulase-negative staphylococci. J Clin Microbiol. 2003;41(10):4740-4.

13. Seifi N, Kahani N, Askari E, Mahdipour S, Naderi NM. Inducible clindamycin resistance in Staphylococcus aureus isolates recovered from Mashhad, Iran. Iran J Microbiol. 2012;4(2):82-6. 